\title{
Photothermal Lensing Signal Enhancement by the Transient Absorption of Photoexcited States in Liquid Solutions
}

\author{
Akira HaRATA ${ }^{\dagger}$ and Naonori YAMAgUCHI \\ Department of Molecular and Material Sciences, Graduate School of Engineering Sciences, Kyushu University, \\ Kasugakoen 6-1, Kasuga, Fukuoka 816-8580, Japan
}

\begin{abstract}
A two-color double-pulse excited photothermal lensing measurement was performed to investigate a signal enhancement owing to transient absorption by the excited states of solute molecules in liquid solutions. A colinear, mode-mismatched configuration with a continuous-wave probe laser at $633 \mathrm{~nm}$ was adapted with a focused pump pulse $(6.9 \mathrm{~ns}, 355 \mathrm{~nm})$ and a sequentially incident softly focused second-pump pulse $(7.2 \mathrm{~ns}, 532 \mathrm{~nm})$. The signal-intensity dependence on the energies of the pump and the second-pump pulses was measured for $1.0 \mu \mathrm{M}$ solutions of $2,2^{\prime}-p$-phenylenebis $(5-$ phenyloxazole) in 1-butanol and anthracene in ethanol. A signal enhancement was observed for both solutions. The signal intensity under certain conditions was one order higher than that expected from ground-state absorption. A discussion was made on determination of the absorption coefficient of the transient species and on the absorption saturation of the ground and excited states.
\end{abstract}

(Received February 26, 2000; Accepted April 26, 2000)

\section{Introduction}

A photothermal lensing (PTL) measurement is one of the most sensitive methods for detecting chemical species, especially non-luminescent substances, in both gas and condensed phases. This method has been used as a suitable investigating tool in a wide range of application fields. ${ }^{1}$ For photothermal methods like photoacoustic spectroscopy, PTL spectroscopy, photothermal deflection spectroscopy, and others, the signal magnitude is directly related to the temperature rise in a light-illuminated material and to the amount of heat generated via optical absorption and subsequent nonradiative relaxation. One of the main factors resulting in good sensitivity is that stronger excitation light causes a larger heat-generation rate. Another factor is that heat accumulation for longer time results in a larger temperature rise. In addition, PTL methods are effective for small volumes of heat accumulation, because the spatial gradient of the refractive index, which is coupled to the temperature gradient by the thermooptic-coupling constant, influences the sensitivity. Crossed-beam and colinear configurations have been applied to probe small volumes. The detection limit of target molecules in liquid solution at room temperature has been improved to the level of one hundred molecules in $200 \mathrm{fL}^{2}$ and in $1.3 \mathrm{fL}^{3}$ and less than one molecule in $3 \mathrm{fL}^{4}$ of the probe volume. However, molecules in a liquid move around so fast that these values of the molecule numbers are time-averaged. Single-molecule detection in liquid solutions, where molecules are counted one by one, has been realized by means of fluorescence measurements. ${ }^{5,6}$ As for nonfluorescent substances in a liquid, the counting of single colloidal silver particles with a diameter of $10 \mathrm{~nm}$ has been carried out by PTL measurements under a microscope. ${ }^{7}$ For nonfluorescent small

† To whom correspondence should be addressed.

E-mail: harata@mm.kyushu-u.ac.jp molecules in solution, electrochemical methods can detect single molecules. ${ }^{8}$ Surface-enhanced Raman scattering may be applied if the adsorbed molecule on a silver nanoparticle is the target molecule. ${ }^{9}$ However, some technical improvements are still required to count small nonfluorescent molecules in solution.

Generally, the PTL signal of a dilute solution linearly increases with the excitation light intensity, but under a focused laser beam, the saturation of optical absorption easily takes place and limits the PTL-signal increase, even if the excitation intensity increases. The absorption saturation, settling the upper limit of the photo-induced heat generation rate per single target molecule, limits the sensitivity of detection. To obtain a large PTL signal beyond the limitation, the use of transient absorption (optical absorption by molecules in photoexcited states) can be the best method. Heat generation via transient absorption has been observed and applied to investigate the dynamics of molecules in dilute solution, where sequential pulsed excitation was used in combination with photothermal detection. ${ }^{10-13}$ For transient absorption, high efficiency is expected of photon energy conversion into heat, because highly excited molecules in liquid rapidly and nonradiatively relax to the lowest vibrational level of the lowest electronic excited state. Moreover, multiple roundtrips in molecular states between the lowest electronic excited level and a higher level may occur during light illumination for the target molecules in a liquid solution. For molecular solids, the roundtrips are known to be one of the important mechanisms of optical ablation under highintensity laser illumination, where extremely large energy is optically deposited in the material at a time. ${ }^{14}$

In this work, we studied PTL-signal enhancement using transient absorption, by the two-color sequential double-pulsed excitation of solute molecules in liquids. It was found that the signal intensity under certain conditions was one order higher than that expected from the ground-state absorption. First, a theoretical explanation for the PTL-signal enhancement is described, while discussing a mode-mismatched colinear-type 
configuration of three laser beams focused on the sample to relate the transient absorption coefficient to the magnitude of the signal enhancement. The experimental details and results are given next.

\section{Theory}

\section{Saturation of PTL signal by optical absorption saturation}

Under intense laser irradiation, the absorption coefficient $(\alpha)$ is known to depend on the light intensity $(I)$ as $\alpha(I)=\alpha_{0} /(1+$ $I / I_{\mathrm{s}}$ ), where $\alpha_{0}$ is the unsaturated absorption coefficient. The absorption saturation threshold $\left(I_{\mathrm{s}}\right)$ indexes when the saturation becomes serious, and is given by $I_{\mathrm{s}}=h v / 2 \sigma \tau_{\text {eff }}$, where $h v$ is the photon energy, $\sigma$ is the absorption cross-section of the molecule, and $\tau_{\text {eff }}$ denotes the average value of the photoexcited state lifetime during the irradiation. ${ }^{15}$ The absorption saturation is significant for focused pulsed-laser beam irradiation (duration $t_{\mathrm{p}}$ and radius $w$ ), even if the energy per pulse $(E)$ is small, because $I=E / \pi w^{2} t_{\mathrm{p}}$ is large.

In a material under focused pulsed-laser irradiation, a part of the light energy is converted into heat, causing the temperature to jump at the laser spot. A refractive-index gradient, which mimics the temperature gradient, is formed around the spot and works as a lens element. The lens-like element (thermal lens) is detectable with a probe beam passing through the spot by monitoring the change in the divergence. ${ }^{1}$ With a well-arranged experimental setup, the PTL-signal intensity $(Q)$ has a linear relation with the amount of photo-generated heat, which is proportional to $\alpha E$ under a small-absorption condition, i.e., $\alpha$ $\ll 1 \mathrm{~cm}^{-1}$. The above relations provide the following equation for PTL-signal saturation:

$$
Q=Q_{\infty} E /\left(E+E_{\mathrm{s}}\right),
$$

where $E_{\mathrm{s}}\left(=\pi w^{2} t_{\mathrm{p}} I_{\mathrm{s}}\right)$ is the pulse energy at the saturation threshold. It is obvious that $Q$ cannot exceed a finite value $\left(Q_{\infty}\right)$ even at large $E$. Namely, the PTL signal suffers saturation under high-intensity excitation.

\section{PTL signal for a mode-mismatched co-linear configuration of three laser beams}

There are a variety of experimental setups for PTL-signal measurements. ${ }^{1,16}$ PTL-signal enhancement is commonly expected whenever transient absorption is utilized. However, for convenience, we present below a certain PTL setup of a mode-mismatched co-linear configuration. We consider three laser beams of different wavelengths (ultraviolet, green, and red), different diameters (large, small, and large) and different types (pulse, pulse, and continuous wave). These beams are identified as pump, second-pump, and probe beams, respectively. When all of the beams propagate along the same axis ( $z$-axis) and are focused by a convex lens into a sample cell, the beam paths near their focal points are schematically sketched in Fig. 1, where thick, dotted, and thin curves represent the pump, secondpump, and probe beams, respectively. The beam propagation is perpendicular to the $x-y$ plane, and the origin of the system (o) is placed at the focus of the probe (red) beam. The PTL effect induced by the pump beam is monitored with the probe beam. Transient absorption by the second-pump beam, incident after the pump beam, causes the PTL-signal enhancement.

A chromatic aberration of the convex lens makes the three beams mode-mismatched. In other words, the positions of their focal points are different, and they generally have different beam sizes at the focal points. The configuration shown in Fig.

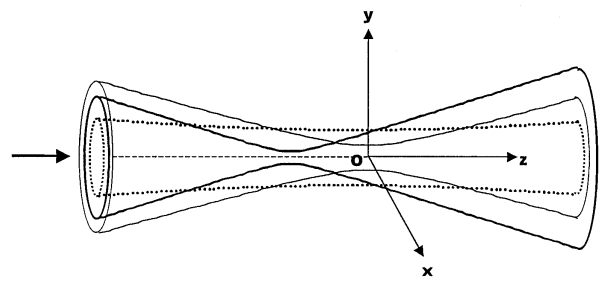

Fig. 1 Schematic illustration showing the overlap of three beams with different wavelengths, convergences, and focus positions, near their focuses.

1 results from the fact that larger diameter beams at the front of the convex lens are more tightly focused and shorter wavelength beams have focal points closer to the lens and smaller diameters. The point of this beam configuration is such that the second-pump beam has a sufficiently small divergence and a sufficiently large radius to provide nearly homogenous illumination around the focal point of the pump beam. The spatial distribution of the heat source by the pump beam has the same shape as that caused by the transient absorption of the second-pump beam, because the distribution of the photoexcited species has the same shape as the heat source.

When a small-area photodiode is used to measure the probebeam intensity at a position on the axis $(x=y=0)$ and at a large $z$, the PTL effect is observed as an increase in the photocurrent intensity. This is understandable for a thin sample, in which the intensity increase is proportional to $-z_{0}(\mathrm{~d} n / \mathrm{d} T)\left(\partial^{2} T / \partial r^{2}\right)_{r=0}$, where $z_{0}(<0)$ is the position of the sample, $n$ is the refractive index, $T$ is the temperature, and $r$ is the distance from the $z$ axis. ${ }^{1}$ Even for the thick sample considered for the configuration above, we can conclude that most of the PTL effect is caused in the region near to the focal point of the pump beam, where photo-induced $\left(\partial^{2} T / \partial r^{2}\right)_{r=0}$ is the largest and $z_{0}$ has a non-zero value. Although the second-pump beam, itself, may generate heat to cause the PTL effect by ground-state absorption, the spatial distribution of the heat is different from that by the pump beam. A relatively small contribution is expected because the size of the beam is large.

\section{Signal enhancement by transient absorption}

Here, we consider the PTL signal under independent or sequential irradiation to the sample by the pump and secondpump pulses. The independent irradiation of the pump (secondpump) light generates a PTL signal of magnitude $Q_{1}\left(Q_{2}\right)$ by ground-state absorption. If the second-pump beam irradiates after the pump beam, the magnitude of the PTL signal $(Q)$ would be

$$
Q=Q_{1}+Q_{2}+Q_{12}
$$

where $Q_{12}$ represents the contribution of the transient absorption to the signal. The gain of the PTL signal by the second-pump beam is important for the practical purpose of sensitive detection with species selectivity. We define the gain as

$$
\text { Gain }=Q /\left(Q_{1}+Q_{2}\right) \text {. }
$$

The gain value is theoretically available in a simple way when the absorption saturation effect is neglected. Consider a sample solution with a solute concentration of $C(\mathrm{M})$ and a molar absorption coefficient of the solute $\varepsilon_{i}\left(\mathrm{~cm}^{-1} \mathrm{M}^{-1}\right)$. Subscript $i$ of 1 or 2 is used to represent the pump or the second-pump beam, respectively. The sample is irradiated by light pulses with pulse 
energy $E_{i}(\mathrm{~mJ} / \mathrm{pulse})$, beam radius $w_{i}\left(\mathrm{~cm}^{2}\right)$, and temporal shapes $g_{i}(t)\left(\mathrm{s}^{-1}\right)$ with $\int_{-\infty}^{\infty} g_{i}(t) \mathrm{d} t=1$. When the efficiency $\left(\eta_{i}\right)$ of the light energy converting into thermal energy is considered, the magnitude of PTL signal by single-beam irradiation is expressed as

$$
Q_{i}=G A_{i} \eta_{i} E_{i} w_{i}^{-2} \varepsilon_{i} C,
$$

where $G$ is the gain factor of the electronics. $A_{i}\left(\mathrm{~cm}^{3} / \mathrm{mJ}\right)$ is a proportionality constant which depends on the thermoopticcoupling constant $(\mathrm{d} n / \mathrm{d} T)$ and the geometrical configuration between the $i$-beam, the probe beam, and the sample. Generally, $A_{1}$ is unequal to $A_{2}$ because of the different geometry.

When the second-pump beam irradiates the sample just after pulsed illumination of the pump beam, the time-dependent concentration of the photoexcited molecule $C_{\mathrm{ex}}(t)(\mathrm{M})$ should be considered, and we obtain

$$
Q_{12}=G A_{1} \eta_{12} E_{2} w_{2}{ }^{-2} \varepsilon_{12} \int_{-\infty}^{\infty} C_{\mathrm{ex}}(t) g_{2}(t) \mathrm{d} t,
$$

where $\varepsilon_{12}\left(\mathrm{~cm}^{-1} \mathrm{M}^{-1}\right)$ is the molar-absorption coefficient for the transient absorption and $\eta_{12}$ is the efficiency of light energy converting into thermal energy for the relaxation process from a highly photoexcited state to the lowest electronic photoexcited state. We can use the proportionality constant $\left(A_{1}\right)$ in Eq. (5) because of the reason discussed before. Although the concentration of the photoexcited molecules is a function of space, no generality is lost when we use values on the beam axis and at the pump-beam waist to calculate the concentration. At this point, the light intensity of the pump pulse is given by $2 E_{1} g_{1}(t) / w_{1}^{2}\left(\mathrm{~mW} / \mathrm{cm}^{2}\right)$, and if the excited state has a single lifetime of $\tau$, then

$$
C_{\text {ex }}(t)=B_{1} E_{1} w_{1}^{-2} \varepsilon_{1} C \int_{-\infty}^{t} g_{1}\left(t^{\prime}\right) e^{-\left(t-t^{\prime}\right) / \tau} \mathrm{d} t^{\prime},
$$

where $B_{1}=2 \times 10^{3} \ln (10) /\left(\pi N_{\mathrm{A}} h v_{1}\right)\left(\mathrm{M} \mathrm{mJ}^{-1} \mathrm{~cm}^{3}\right), h v_{1}(\mathrm{~mJ})$ is the photon energy of the pump pulse, and $N_{\mathrm{A}}$ is Avogadro's number. We can use Eqs. (2) - (6) to obtain the relations

$$
\frac{Q-Q_{2}}{Q_{1}}=1+\chi \frac{\eta_{12} \varepsilon_{12}}{\eta_{1}} E_{2},
$$

and

$$
\text { Gain }=1+\frac{1}{1+Q_{2} / Q_{1}} \chi \frac{\eta_{12} \varepsilon_{12}}{\eta_{1}} E_{2},
$$

where $\chi\left(\mathrm{M} \mathrm{cm} \mathrm{mJ}^{-1}\right)$ is a proportionality constant given by

$$
\chi=B_{1} w_{2}{ }^{-2} \int_{-\infty}^{\infty} \mathrm{d} t \int_{-\infty}^{t} \mathrm{~d} t^{\prime} \times g_{2}(t) g_{1}\left(t^{\prime}\right) e^{-\left(t-t^{\prime}\right) / \tau} .
$$

The value of $\chi$ can be calculated for a given experimental condition. $\eta_{1}$ is a known value for the ground state that is estimable from fluorescence quantum efficiency and the Stokes shift in the emission spectrum. ${ }^{17}$ Generally, we can assume $\eta_{12}$ $=1$. Thus, Eq. (7) demonstrates that $\varepsilon_{12}$ can be experimentally determined. Equation (8) indicates that a larger gain can be obtained with stronger $E_{2}$ because $Q_{2} / Q_{1}$ is negligible for most cases of interests to us.

It should be noted that the absorption saturation effect is not taken into account in Eqs. (4) - (9). Some modification is

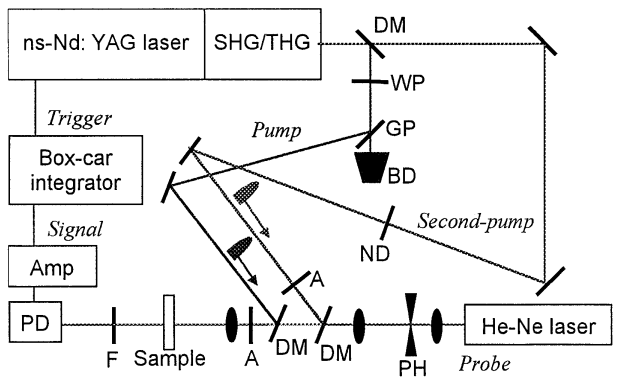

Fig. 2 Schematic illustration of the experimental setup for thermal lensing signal enhancement using transient absorption. A, ails; BD, beam dump; DM, dichroic mirror; F, colored glass filter; GP, glass plate; ND, neutral density filter; PD, photodiode; $\mathrm{PH}$, pinhole; and WP, half-wave plate. A set of WP and GP was used for adjusting pump-beam intensity.

required under the strong pump- or the strong second-pump condition. If the saturation of transient absorption becomes serious at a strong $E_{2}$, the (Gain-1) behaves as the $E$-dependence in Eq. (1). Although the saturation of ground-state absorption can interfere with the gain, it is not very simple to obtain a useful analytical expression of the saturation effect (see Appendix). It is obvious from Eq. (8) that the gain is independent of $E_{1}$ when $Q_{2} / Q_{1}$ is negligible, making the influence of the ground-state absorption saturation to the gain appear in higher order terms.

\section{Experimental}

A schematic illustration of the experimental setup for PTLsignal enhancement using transient absorption is shown in Fig. 2. The third $(355 \mathrm{~nm})$ and second $(532 \mathrm{~nm})$ harmonics of a Qswitched Nd-doped yttrium aluminum garnet laser (Surelite I10, Continuum) was used as pump and second-pump beams, respectively. The pulsed laser was operated at $10 \mathrm{~Hz}$ of repetition frequency. The intensity of the pump beam was adjusted with a half-wave plate and Brewster's angle reflector of the glass plate, and that of the second-pump beam was adjusted with a set of neutral-density filters. The polarization planes of the beams were set parallel to each other. A continuous-wave He-Ne laser (632.8 nm, Model 1101P, Uniphase) was used as a probe beam combined with a spatial filter made up of an objective lens $(\times 10$, NA 0.25$)$, a $25-\mu \mathrm{m}$ pinhole, and a convex lens. These three beams were colinearly combined with two dichroic mirrors after they passed through irises of appropriate sizes. They were focused into a quartz sample cuvette by an achromatic convex lens with a focusing length of $200 \mathrm{~mm}$ (UDL-30-200P, Sigma-Koki). The beam parameters are listed in Table 1 , and a detailed discussion is provided later. The probe-beam intensity on the optical axis was monitored at $500 \mathrm{~mm}$ from the sample cell, with a photodiode connected to an isolation amplifier (P-62, NF circuit design block Co.). In front of the photodiode, a red-colored glass filter (SCF-50S-62R, Sigma-Koki) and an interference filter $(632.8 \pm 5.5 \mathrm{~nm}$, Edmund Scientific) were placed to cut off the pump and second-pump beams. The output showed a transient signal increase and succeeding decay in $20 \mathrm{~ms}$ when the pump pulse illuminated the sample. A box-car integrator (BX-530A, NF circuit design block Co.) was used to hold the peak intensity. The accumulated box-car output was recorded as the PTL-signal intensity. The temporal shapes of the optical 
Table 1 The laser-beam parameters of the present experiment using the focusing lens (UDL-30-200P, Sigma-Koki)

\begin{tabular}{|c|c|c|c|c|c|c|c|}
\hline & \multirow{2}{*}{$\begin{array}{l}\text { Wavelength/ } \\
\mathrm{nm}\end{array}$} & \multirow{2}{*}{$\begin{array}{c}\text { Focal length } / \\
\text { mm }\end{array}$} & \multicolumn{3}{|c|}{ Diameter/ $\mu \mathrm{m}$} & \multirow{2}{*}{$\begin{array}{l}\text { Confocal } \\
\text { length } / \mathrm{mm}\end{array}$} & \multirow{2}{*}{$\begin{array}{c}\text { Pulse width } \\
\text { ns }\end{array}$} \\
\hline & & & $\begin{array}{l}\text { at entrance } \\
\text { apertures }\end{array}$ & at beam waists ${ }^{\mathrm{b}}$ & $\begin{array}{l}\text { at the pump } \\
\text { beam waist } \mathrm{t}^{\mathrm{b}}\end{array}$ & & \\
\hline Pump & 355 & 204.9 & 6500 & 14.2 & 14.2 & 0.45 & 6.9 \\
\hline 2nd-pump & 532 & 206.6 & 2300 & 60.8 & 63.9 & 5.47 & 7.2 \\
\hline Probe & 632.8 & 206.9 & 6000 & 27.8 & 64.3 & 0.96 & $(\mathrm{CW})$ \\
\hline
\end{tabular}

a. Calculated with reference data in Ref. 18. b. Calculated by assuming Gaussian laser beams. c. Measured values of full-width at the half-maximum.
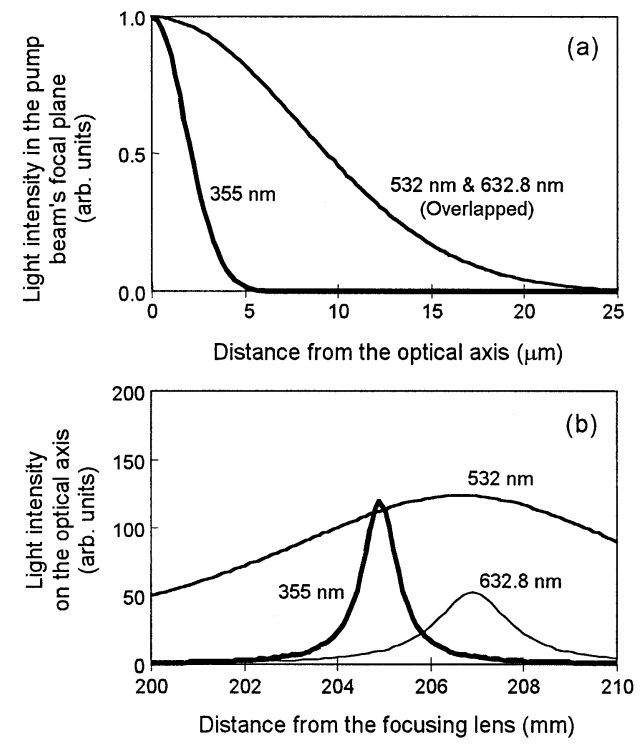

Fig. 3 Calculated intensity distribution of the three laser beams (a) in the focal plane of the 355-nm pump beam as a function of distance from the optical axis; and (b) on and along the optical axis.

pulses were measured with a fast PIN photodiode and a 500MHz digitizing oscilloscope (TDS-520C, Tektronix).

The samples used were a 1-butanol solution of 2,2'-pphenylenebis(5-phenyloxazole) (POPOP) and an ethanol solution of anthracene. The typical concentration was $1.0 \mu \mathrm{M}$. The absorption coefficient at $355 \mathrm{~nm}$ was $0.02 \mathrm{~cm}^{-1}$ and that at $532 \mathrm{~nm}$ was negligibly small for both $1.0 \mu \mathrm{M}$ POPOP and 2.5 $\mu \mathrm{M}$ anthracene solutions. Anthracene, ethanol, and 1-butanol of special grade and POPOP for scintillation were purchased from Kishida Chemical Co., Ltd. and used without further purification.

\section{Results and Discussion}

Characteristics of the laser beams in the PTL measurement

Because the PTL signal in the two- and three-color experiments strongly depends on the spatial overlaps of the laser beams, it is important to specify the beam parameters (position of focus and beam diameters at certain positions) for each laser beam before discussing any experimental results. Because the well-characterized convex lens of UDL-30-200P was used to focus the laser beams with small divergence, the positions of the focuses were estimated with values in the characteristic data table ${ }^{18}$ of the wavelength-dependent focal length of the lens. We calculated the beam radius at certain positions (Table 1) and intensity distributions (Fig. 3). We assumed that all beams had Gaussian intensity distributions in their cross-sections, and that their diameters at the lens were the
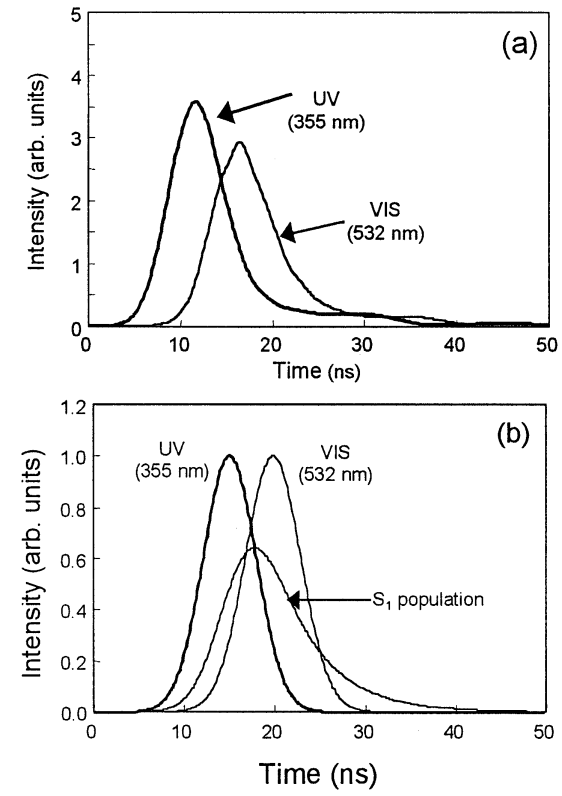

Fig. 4 (a) Measured transient waveforms of the pulsed-laser beams: accumulation was for 512 pulses; delay time of the visible light was $4.8 \mathrm{~ns}$ with respect to the ultraviolet light. (b) Transient waveforms calculated as Gaussian temporal profiles separated by $4.8 \mathrm{~ns}$. The population transient of the $S_{1}$-state with $5.0 \mathrm{~ns}$ of lifetime excited by the ultraviolet light pulse is also shown.

same sizes as those of the entrance apertures. Because $\left(\partial^{2} T / \partial r^{2}\right)_{r=0}$ is important for the PTL effect, most of the effect is generated in the region where the pump-beam intensity is large, namely $204-206 \mathrm{~mm}$ from the focusing lens. We confirmed that the 532-nm second-pump beam would have nearly homogeneous intensity in the paraxial region around the focal point of the 355-nm pump beam, as shown in Figs. 3 (a) and (b).

Figure 4(a) shows the temporal shapes of pulsed beams averaged over 512 pulses for both the $355-\mathrm{nm}$ pump pulse and the 532-nm second-pump pulse. Although both pulses had fluctuating multiple peaks separated by a 2 -ns time interval when we observed the shapes of single pulses, the averaged shapes were in a Gaussian-like distribution with weak tails. The temporal shapes were well expressed as

$$
g_{1}(t)=\sqrt{\frac{2}{\pi}} \frac{1}{\tau_{1}} \exp \left[-2\left(t / \tau_{1}\right)^{2}\right]
$$

and

$$
g_{2}(t)=\sqrt{\frac{2}{\pi}} \frac{1}{\tau_{2}} \exp \left[-2\left\{\left(t-t_{\mathrm{d}}\right) / \tau_{2}\right\}^{2}\right]
$$




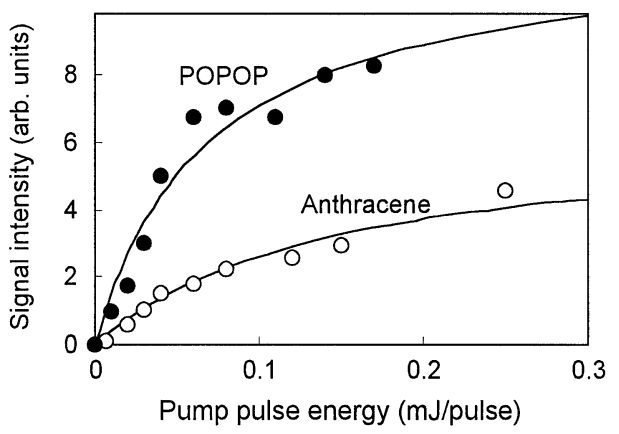

Fig. 5 Pump-pulse energy dependence of thermal lensing signal intensity for $1.0 \mu \mathrm{M}$ solutions of POPOP in 1-butanol and anthracene in ethanol. The solid curves are fitting results showing saturation thresholds of $0.068 \mathrm{~mJ} /$ pulse and $0.15 \mathrm{~mJ} /$ pulse for POPOP and anthracene, respectively.

where $t_{\mathrm{d}}$ is the delay time of the second pulse with respect to the pump pulse, and $\tau_{1} \sqrt{2 \ln 2}\left(\tau_{2} \sqrt{2 \ln 2}\right)$ is the full width at the half-maximum of the pump (second-pump) pulse. The pulse widths were calculated by a fitting procedure, and are listed in Table 1. The value of $t_{\mathrm{d}}$ was $4.8 \mathrm{~ns}$. In Fig. 4(b), the normalized shapes of the Gaussian beams are shown together with the calculated population transient of the lowest photoexcited singlet state $\mathrm{S}_{1}$ with a lifetime of $5.0 \mathrm{~ns}$ as an example. The proportionality constant $(\chi)$ is dominated by the overlap between the second-pump pulse and the $S_{1}$ population. It is apparent that by changing the timing delay, we can optimize the large gain, although we did not do so in the present experiment. The proportionality constant $(\chi)$ was calculated with reference values having lifetimes of $1.3 \mathrm{~ns}$ for POPOP in ethanol ${ }^{19}$ and 5.0 ns for anthracene in ethanol ${ }^{19,20}$ to be $3.6 \times 10^{-2}$ and $1.4 \times 10^{-1}$ $\left(\mathrm{M} \mathrm{cm} \mathrm{mJ} \mathrm{m}^{-1}\right)$, respectively. These values are used in a later discussion.

\section{Saturation of the PTL signal by the pump pulse}

The pump-pulse energy dependence of the PTL-signal intensity is shown in Fig. 5 for both the $1.0 \mu \mathrm{M}$ POPOP and 1.0 $\mu \mathrm{M}$ anthracene solutions. The saturation of optical absorption can easily result from the pulsed excitation of a strong absorption band, and the dependence tends to deviate from a linear relation. The deviation was attributed to absorption saturation because the absolute signal intensity was small enough to neglect the intrinsic nonlinear behavior of the PTL signal (the change in the probe light intensity detected by the photodiode was less than $2.4 \%$ of the unperturbated intensity, even at the maximum PTL-signal intensity). The solid curves in Fig. 5 provide the best fitting to Eq. (1). The saturation threshold $\left(E_{\mathrm{s}}\right)$ was determined to be $0.068 \mathrm{~mJ}$ for POPOP and $0.15 \mathrm{~mJ}$ for anthracene. These values corresponded to $0.59 \mathrm{ps}$ and 0.68 ps of $\tau_{\text {eff }}$, respectively, where the $\sigma$ used were $7.7 \times$ $10^{-17} \mathrm{~cm}^{2}$ for POPOP and $3.1 \times 10^{-17} \mathrm{~cm}^{2}$ for anthracene. Although these $\tau_{\text {eff }}$ values were much smaller than the fluorescence lifetimes of either compound, they are not

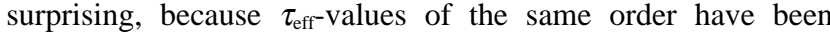
reported for a dye in poly(methyl methacrylate) by near-infrared absorption. ${ }^{15}$ Any inaccuracies in the beam size and pulse width could have some influence on the quantitative evaluation of $\tau_{\text {eff }}$ The spatial distribution of the beam intensity and the temporal shapes of the light pulse could cause an underestimation of $\tau_{\text {eff }}$ Besides these, it is not easy to provide a theoretical explanation for a discrepancy of three orders of magnitude, even when applying a complex model of dynamics that takes the

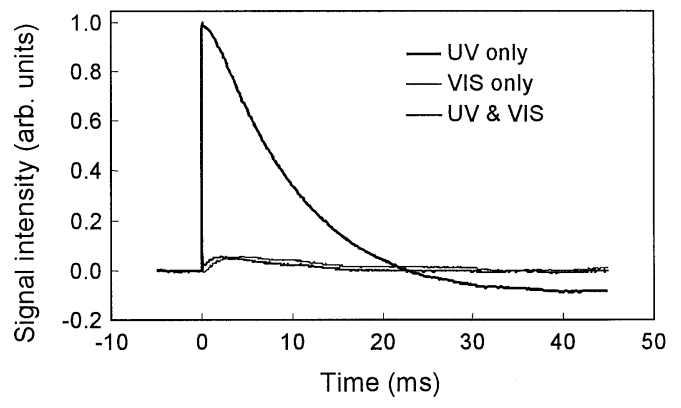

Fig. 6 Enhancement of thermal lensing signal by sequential incidence of pump and second-pump pulses. Transient waveforms pumped by ultraviolet pulse only, by visible pulse only and by both ultraviolet and visible pulses are shown. The sample was $1.0 \mu \mathrm{M}$ POPOP solution in 1-butanol.

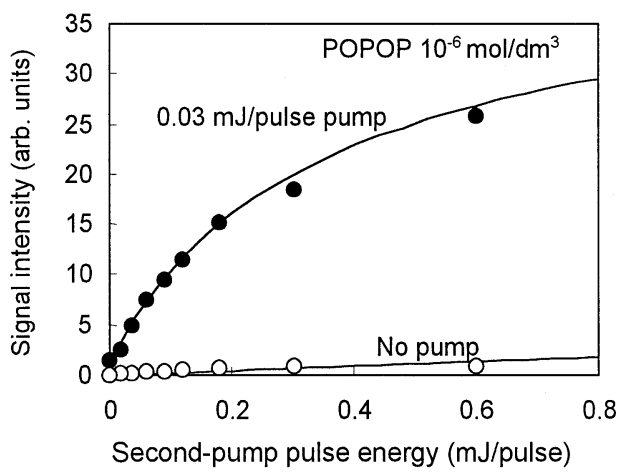

Fig. 7 Second-pump pulse energy dependence of thermal lensing signal intensity for $1.0 \mu \mathrm{M}$ POPOP solution in 1-butanol. The solid curve for the $0.03-\mathrm{mJ} /$ pulse pump condition is a fitting result showing an absorption saturation threshold of $0.31 \mathrm{~mJ} / \mathrm{pulse}$. The solid line for the no-pump condition is the result of linear regression.

degeneracy of the ground and excited states into account. However, it is sufficient here to note that the PTL signal showed saturation when the pump laser intensity increased, and that signal enhancement by transient absorption is one possible way to overcome the saturation limit.

\section{Enhancement of the PTL signal by the second-pump pulse}

Signal enhancement by the second-pump pulse is clearly demonstrated in Fig. 6. Typical traces of PTL-signal transients are shown for the $1.0 \mu \mathrm{M}$ POPOP solution. The POPOP dye has strong optical absorption at $355 \mathrm{~nm}$ and weak absorption at $532 \mathrm{~nm}$. A larger enhancement was achieved with an intense 532-nm pulse following a weak 355-nm pulse, while independent irradiation of the $532-\mathrm{nm}$ pulse or the $355-\mathrm{nm}$ pulse generated small signals. The PTL-signal intensity depended on the second-pump pulse energy, as shown in Fig. 7, while the pump-pulse energy was fixed to $0.03 \mathrm{~mJ} / \mathrm{pulse}$. The signal obtained with no pump pulse was due to weak absorption at $532 \mathrm{~nm}$. The signal intensity at zero of the second-pump pulse energy was caused by the pump beam at $355 \mathrm{~nm}$. If there were no transient absorption at $532 \mathrm{~nm}$, a simple summation of signal intensities should be obtained with the two-color excitation. A much larger signal observed was attributed to transient absorption by photoexcited molecules.

The transient absorption of POPOP also showed a saturation behavior, as in Fig. 7. The solid curve in Fig. 7 shows the best fitting to Eq. (1), with which we estimated the saturation 


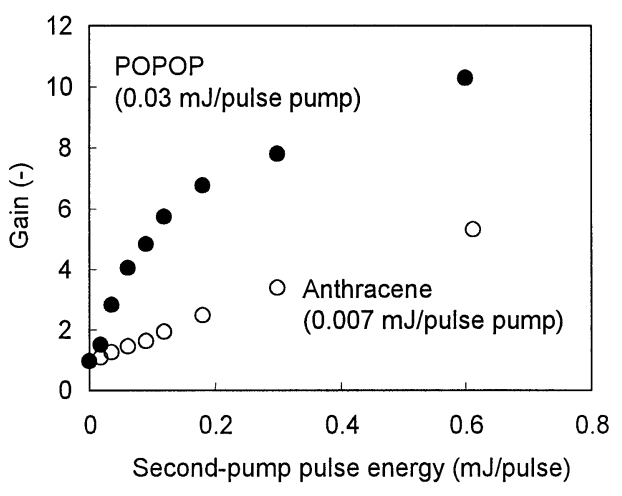

Fig. 8 Second-pump pulse energy dependence of gain in the thermal lensing signal intensity for $1.0 \mu \mathrm{M}$ solutions of POPOP in 1butanol and anthracene in ethanol. Pump-pulse energy was 0.03 $\mathrm{mJ} / \mathrm{pulse}$ for POPOP and $0.007 \mathrm{~mJ} /$ pulse for anthracene, respectively.

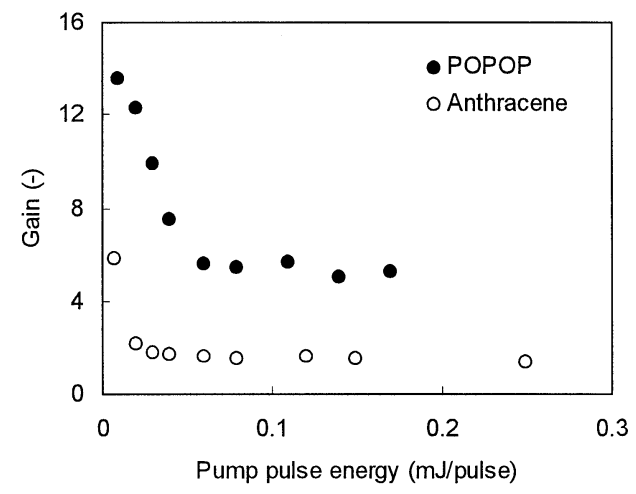

Fig. 9 Pump-pulse energy dependence of gain in the thermal lensing signal intensity for $1.0 \mu \mathrm{M}$ solutions of POPOP in 1-butanol and anthracene in ethanol. Second-pump pulse energy was 0.60 $\mathrm{mJ} /$ pulse for POPOP and $0.62 \mathrm{~mJ} / \mathrm{pulse}$ for anthracene, respectively. threshold of transient absorption to be $0.31 \mathrm{~mJ}$. With the molar absorption coefficient, $\varepsilon_{12}=7.5 \times 10^{3}\left(\mathrm{~cm}^{-1} \mathrm{M}^{-1}\right)^{21}$ for a transient absorption of POPOP at $532 \mathrm{~nm}$, we estimated $\tau_{\text {eff }}=4.7 \mathrm{ps}$. This value was 8 times larger than that of ground-state absorption saturation. POPOP dyes are excited to the $S_{1}$ states by $355-\mathrm{nm}$ light ${ }^{22}$ and the molecules in $S_{1}$ states are excited to the $\mathrm{S}_{3}$ states by 532-nm light. ${ }^{22}$ Although the lifetime of the highly excited state is unknown for POPOP, it is generally expected to be less than several picoseconds for such dye molecules in a liquid solution at room temperature. We concluded that there is no simple relation between the $\tau_{\text {eff }}$ values and the lifetimes of the ground and excited states, and that it is necessary to evaluate the saturation threshold experimentally, although the saturation threshold is one of the decisive factors to achieve a higher gain.

Figure 8 shows the gain dependence on the second-pump pulse energy. Note that first, the gain was over 10 for POPOP under an appropriate condition, and second, the gain depended on the kind of molecular species. These results are suggestive when ultra-sensitive and species-selective detection is conducted for the ultratrace analysis of a sample in a condensed phase. A larger gain is expected as the second-pump pulse energy increases. Besides absorption saturation, the gain will be limited by some nonlinear optical phenomena, such as the optical breakdown of a solution (or cell wall) or stimulated Raman effects of the solvent, but the experimental conditions can be optimized to achieve high sensitivity. The data plots in Fig. 8 for anthracene pumped with $0.007 \mathrm{~mJ}$ at $355 \mathrm{~nm}$ are on a straight line up to $0.62 \mathrm{~mJ}$ of second-pump pulse energy. Since $Q_{2} / Q_{1}$ was negligible for anthracene, the linear dependence is that expected from Eq. (8). The molecular properties of anthracene and the experimental conditions would cause good linearity based on the rapid depletion of molecules in the excited singlet state and have no influence on the triplet- and ground-state absorption at the second-pump wavelength. Anthracene is a molecule with a high triplet-formation quantum yield of 0.7 in ethanol. ${ }^{23}$ The forbidden transition form the singlet ground state to the lowest excited triplet state around 550 $\mathrm{nm}$ has an absorption coefficient smaller than $10^{-4}\left(\mathrm{~cm}^{-1} \mathrm{M}^{-1}\right) .^{24}$ Small transient absorption is expected at $532 \mathrm{~nm}$ by triplettriplet transitions that lie above $520 \mathrm{~nm}$ of photon energy in polymethyl-methacrylate ${ }^{25}$ and around $430 \mathrm{~nm}$ and above 460 $\mathrm{nm}$ of photon energy in cyclohexane and benzene. ${ }^{26}$ The lifetime of the triplet state is much shorter than $100 \mathrm{~ms}$ of the repetition rate. For POPOP, a linear dependence of the gain was observed only for small values of the second-pump pulse energy, and the gain was saturated for large energy. The slope of the initial linear portion was $46\left(\mathrm{~mJ}^{-1}\right)$. The slope of the line for anthracene was $7.3\left(\mathrm{~mJ}^{-1}\right)$.

We estimated $\eta_{1}$ of 0.20 and 0.81 for POPOP and anthracene, respectively. ${ }^{27}$ These values correspond to $\chi \eta_{12} / \eta_{1}$ of $1.8 \times 10^{-1}$ $\left(\mathrm{M} \mathrm{cm} \mathrm{mJ}^{-1}\right)$ for both POPOP and anthracene. From the slope of the gain curve, $\varepsilon_{12}$ was estimated to be $2.6 \times 10^{5}\left(\mathrm{~cm}^{-1} \mathrm{M}^{-1}\right)$ for POPOP and $4.1 \times 10^{4}\left(\mathrm{~cm}^{-1} \mathrm{M}^{-1}\right)$ for anthracene. The experimental value of $\varepsilon_{12}$ for POPOP was 35-times larger than the reference value, $7.5 \times 10^{3}\left(\mathrm{~cm}^{-1} \mathrm{M}^{-1}\right)$, at $532 \mathrm{~nm}$. Unfortunately, we could find no direct reference of $\varepsilon_{12}$ for anthracene. The $\varepsilon_{12}$ value was estimated to be $2 \times 10^{3}\left(\mathrm{~cm}^{-1}\right.$ $\mathrm{M}^{-1}$ ) at $532 \mathrm{~nm}$, the same value as for 9,10-diphenyl-anthracene, because the shapes of the transient absorption spectra between 500 and $600 \mathrm{~nm}$ were similar for both molecules with the same chromophore. $^{21,28}$ The value $\varepsilon_{12}=4.1 \times 10^{4}\left(\mathrm{~cm}^{-1} \mathrm{M}^{-1}\right)$, which was determined experimentally, is significantly large. It is possible to underestimate $\chi$ by a possible inaccuracy in $\omega_{2}$, although, to compensate the 35-times discrepancy, we need the implausible assumption of a 5.9-times larger $\omega_{2}$. A large $\eta_{1}$ can somewhat lessen the discrepancy, but it seems that multipletime photoexcitation of certain molecules in the photoexcited states took place to enlarge the effective $\varepsilon_{12}$.

Figure 9 shows the pump-pulse energy dependence of the gain, where the second-pump pulse energy was fixed to $0.6 \mathrm{~mJ}$. The gain increased as the pump intensity decreased. It gradually decreased with increasing pump-pulse energy, and at high pump conditions the gain was 5.4 and 1.5 for POPOP and anthracene, respectively. The pump-intensity-dependent behavior of the gain curves is beyond the model described before. It might be considered that the behavior is caused by the pump-pulse energy-dependence of $\chi$, because under strong pump conditions the time-evolution of the photoexcited species concentration would be different from that under weak pump conditions. Another possible reason could be found in $\eta_{1}$ under the strong pump condition. However, no conclusive reasons for the mechanisms were found, even with a modified model given in Appendix, and further investigation is required. It is suggestive that the pump-pulse energy above which the gain achieved a constant value corresponded to the saturation threshold of the ground-state absorption for POPOP. This seems reasonable in view of the fact that, above the saturation threshold, the population of excited-state molecules does not change appreciably. However, for anthracene, whose yield of 
intersystem crossing is $0.7,{ }^{23}$ a simple explanation, such as that for POPOP above, is inappropriate because the triplet-state formation causes a rapid depletion of the ground-states population.

It is true that mechanisms with higher complexity than our simple theoretical model may explain the PTL-signal enhancement better, but our model is a good guide for designing a sensitive and selective detection system. A preliminary experiment of the PTL measurements in a capillary cell with $0.2-\mathrm{mm}$ optical path length successfully showed signal enhancement of the same order of magnitude and of the same species-dependency. We think that the use of continuous-wave lasers for the pump and second-pump beams is preferable for PTL signal enhancement because optical damage of the cell wall easily takes place for the pulsed laser beams, especially for the capillary cell. Even when focused continuous-wave laser beams are used for excitation, the absorption saturation can be serious for the PTL signal, and our model is applicable with some modification.

\section{Conclusion}

A two-color sequential double-pulse excited PTL measurement was performed to investigate any signal enhancement owing to transient absorption by excited solute-molecules in liquid solutions. A colinear, mode-mismatched configuration was adapted with a focused ultraviolet pump pulse and a sequentially incident softly focused visible pulse designed so as to allow the determination of the effective absorption coefficient of the transient species. A more than one order large signal was obtained in a $1.0 \mu \mathrm{M}$ POPOP solution in hexane, and a speciesselective enhancement was demonstrated. Effects of the absorption saturation for the ground state and the excited state were discussed. The method demonstrated here promises to be crucial for counting non-fluorescent single molecules in liquid solutions and for finding the upper limit in accumulating the photo-induced heat energy during a certain time interval and in a certain space of controlled dimension.

\section{Appendix}

As a modified model explaining PTL-signal saturation caused by optical absorption saturation, we can use a two-level system assuming degeneracy in each level. By considering a constant total concentration of $C=C_{\mathrm{gr}}(t)+C_{\mathrm{ex}}(t)$, the rate equation for the system is given by

$$
\frac{\mathrm{d} C_{\mathrm{ex}}}{\mathrm{d} t}=-\frac{C_{\mathrm{ex}}}{\tau}+B_{1} E_{1} w_{1}^{-2} \varepsilon_{1}\left(C-f C_{\mathrm{ex}}\right) g_{1}(t)
$$

where $C_{\mathrm{gr}}(t)$ is the time-dependent concentration of the molecules in the ground state and $f=1+g_{\mathrm{gr}} / g_{\text {ex }}$ represents the influences of degeneracy in the lower level $\left(g_{\mathrm{gr}}\right.$, ground state) and the upper level ( $g_{\mathrm{ex}}$, excited state). The solution of Eq. (A1) is

$$
\begin{aligned}
C_{\text {ex }}(t)=B_{1} E_{1} w_{1}{ }^{-2} \varepsilon_{1} C \int_{-\infty}^{t} g\left(t^{\prime}\right) \times \\
\exp \left[-\frac{t-t^{\prime}}{\tau}-f B_{1} E_{1} w_{1}^{-2} \varepsilon_{1} \int_{t^{\prime}}^{t} g(\xi) \mathrm{d} \xi\right] \mathrm{d} t^{\prime} .
\end{aligned}
$$

condition. The concentrations of $C$ and $C_{\mathrm{ex}}$ in Eqs. (4) and (5) should be replaced to give the Gain and parameter $\chi$ in Eqs. (7), (8), and (9) under intense pump conditions.

\section{References}

1. S. E. Bialkowski, "Photothermal Spectroscopy Methods for Chemical Analysis", 1996, Wiley, New York.

2. T. G. Nolan and N. J. Dovichi, IEEE Circuites Devices Mag., 1986, 2, 54.

3. K. Sato, H. Kawanishi, M. Tokeshi, T. Kitamori, and T. Sawada, Anal. Sci., 1999, 15, 525.

4. M. Tokeshi, M. Uchida, K. Uchiyama, T. Sawada, and T Kitamori, J. Luminescence, 1999, 83, 261.

5. M. D. Barnes, W. B. Whitten, and J. M. Ramsey, Anal. Chem., 1995, 418A.

6. P. M. Goodwin, W. P. Ambrose, and R. A. Keller, Acc. Chem. Res., 1996, 29, 607.

7. K. Mawatari, T. Kitamori, and T. Sawada, Anal. Chem. 1998, 70, 5037

8. A. J. Bard and F.-R. F. Fan, Acc. Chem. Res., 1996, 29, 572.

9. S. Nie and S. R. Emory, Science, 1997, 275, 1102.

10. M. G. Rockley and J. P. Devlin, Appl. Phys. Lett., 1977, 31 , 24

11. L. J. Rothberg, M. Bernstein, and K. S. Peters, J. Chem. Phys., 1983, 79, 2569.

12. J. M. Heritier and A. E. Siegman, IEEE J. Quantum. Electron., 1983, $Q E-19,1551$.

13. G. G. Yee and D. S. Kliger, 1983, 87, 87.

14. H. Fukumura and H. Masuhara, Chem. Phys. Lett., 1994, $221,373$.

15. S. Chen, I.-Y. S. Lee, W. AA. Tolbert, X. Wen, and D. D. Dlott, J. Phys. Chem., 1992, 96, 7178.

16. M. Franko and C. D. Tran, Rev. Sci. Instrum., 1996, 67, 1.

17. S. E. Braslavski and G. E. Heibel, Chem. Rev., 1992, 92, 1381.

18. The data table is provided by Sigma Koki Co., Ltd. in the catalog for optics and optical coatings.

19. D. M. Rayner, A. E. McKinnon, A. G. Szabo, and P. A. Hackett, Can. J. Chem., 1976, 54, 3246.

20. R. A. Lampert, L. A .Chewter, D. Phillips, D. V. O'Conner, A. J. Roberts, and S. R. Meech, Anal. Chem., 1983, 55, 68.

21. D. Magde, S. T. Gaffney, and B. F. Campbell, IEEE J. Quantum. Electron., 1981, QE-17, 489.

22. Y. V. Rao, P. Venkateswarlu, M. C. George, H. Jagannath, and G. Chakrapani, Spectrochim. Acta, 1988, 44A, 131.

23. D. Lynch and J. F. Endicott, Inorg. Chem., 1988, 27, 2181.

24. N. J. Turro, "Modern Molecular Photochemistry", 1978, Benjamin/Cummings, California, 94.

25. D. Bebelaar, Chem. Phys., 1974, 3, 205.

26. R. Bensasson and E. J. Land, Trans. Farad. Soc., 1971, 67, 1904.

27. To calculate $\eta_{1}$, we referred fluorescence emission spectra in I. B. Berlman, "Handbook of Fluorescence Spectra of Aromatic molecules", 2nd ed., 1971, Academic Press, New York. Values of fluorescence quantum yield were referred from the same book for POPOP and from Ref. 17 for anthracene.

28. C. R. Goldschmit and M. Ottolenhgi, Chem. Phys. Lett., 1970, 4, 570 .

Equation (A2) can be evaluated with the given experimental 